American Journal of Biochemistry and Biotechnology 2 (3): 85-88, 2006

ISSN 1553-3468

(C) 2006 Science Publications

\title{
Hydroxyapatite-induced Phagocytic Activity by Murine Macrophages in vitro
}

\author{
${ }^{1}$ V.K.Gopinath, ${ }^{2}$ M. Musa, ${ }^{1}$ A R. Samsudin, ${ }^{1}$ K. A.M. AI-Salihi and ${ }^{1,2}$ W. Sosroseno \\ ${ }^{1}$ School of Dental Sciences ${ }^{2}$ Department of Immunology \\ School of Medical Sciences, Universiti Sains Malaysia, 16150 Kota Bharu, Malaysia
}

\begin{abstract}
The aim of the present study was to assess phagocytic activities of murine macrophages (RAW264.7 cell line) to hydroxyapatite (HA) particles. Transmission electron microscope (TEM) was used to assess localization of HA particles in the cells at 7, 15, 30 and $60 \mathrm{~min}$. The HA particles and latex beads, as a control, were also incubated with the cells pretreated with various concentration of cytochalasin B or colchicine. Phagocytic index (PI) was used to determine the phagocytic activity at one hr of incubation. TEM analysis showed that HA particles were in direct contact with the cells at 7 min with a few particles located within cell vacuoles. The cells increasingly phagocytosed the particles up to $60 \mathrm{~min}$. Cytochalasin B or colchicine significantly inhibited phagocytic activity of the cells to both HA particles and latex beads in a dose-dependent fashion. Therefore, the results of the present study suggest that HA particles may induce phagocytic activity of RAW264.7cells in an actin and microtubule polymerization-dependent mechanism.
\end{abstract}

Key words: Phagocytic index, RAW264.7 cells, actin, microtubule

\section{INTRODUCTION}

Hydroxyapatite (HA) is used as a bone-replacing biomaterial in reconstructive surgery in view of its encouraging biological properties ${ }^{[1]}$. For example, HA is used as an augmentation material in craniofacial surgery $^{[2]}$ and in the treatment of periodontal defects ${ }^{3]}$.

Following contact between $\mathrm{HA}$ and the surrounding tissues, it may initiate several and complex biological reactions such as inflammatory reaction at the implant site ${ }^{[4]}$. Previous studies in animals indicated activation and ingestion of $\mathrm{HA}$ particles by the macrophages following HA dental implant placement ${ }^{[5]}$, suggesting that contact between HA-macrophages may induce phagocytic activity of these cells to these particles. However, the exact mechanism by which macrophages engulf implanted HA particles, remains to be further investigated. Our previous study showed that HA-induced phagocytosis by RAW264.7 cells may be regulated by nitric oxide ${ }^{[6]}$. Hence the aim of the present study was to assess HA-induced phagocytosis by RAW264.7 cells under Transmission Electron Microscopy (TEM) and to determine the role of actin and microtubule polymerization.

\section{MATERIALS AND METHODS}

Hydroxyapatite (HA) powder (3.5 to $8 \mu \mathrm{m})$ was suspended in sterile saline. Latex beads $(3 \mu \mathrm{m})$ (Sigma, USA) were used as a control. HA particles were stained with crystal violet prior to use for phagocytic assay.

Murine macrophages ( $5 \times 10^{6}$ cells) (RAW 264.7) were incubated with $1 \times 10^{5}$ particles of stained HA or latex beads in $1 \mathrm{ml}$ of culture medium in a sterile tube for one hr. In other experiments, the cells were pretreated with different concentration $(0,1,10$ and 100 $\mu \mathrm{M})$ of cytochalasin $\mathrm{B}$ to inhibit actin polymerization, for $30 \mathrm{~min}$ or colchicine $(0,1,10,20,30$ and $40 \mu \mathrm{g}$ $\mathrm{mL}^{-1}$ ), a microtubule-disrupting agent, for $15 \mathrm{~min}$ prior to incubation with the particles. Cytochalasin B and colchicine were dissolved in sterile saline. Subsequently the cells were washed and then incubated with HA or latex bead. Phagocytic index (PI) was calculated as previously described $^{[6]}$. All materials were purchased from Sigma, USA.

HA-treated cells at all time points with untreated cells as a control were studied under TEM. The samples were prepares as previously described ${ }^{[7]}$. Ultra thin sections were examined by transmission electron microscope (Zeiss, Germany).

Data was analyzed by one-way analysis of variance followed by Fisher's least squared difference were used in the experiments of Cytochalasin B and colchicine treated cells using a statistical package (SPSS, Chicago, IL, USA).

\section{RESULTS}

The cells treated with HA when examined under TEM at 7, 15, 30 and 60 min with untreated cells as control (Fig. 1A). Macrophages showed close direct contact with HA particles at 7 min of incubation (Fig.1B). Few HA particles were seen in the cell cytosol, particularly in phagosome vacuoles.

Corresponding Author: Dr. V.K. Gopinath, School of Dental Sciences, Universiti Sains Malaysia, 16150 Kota Bharu, Malaysia, Fax: 6097642026 

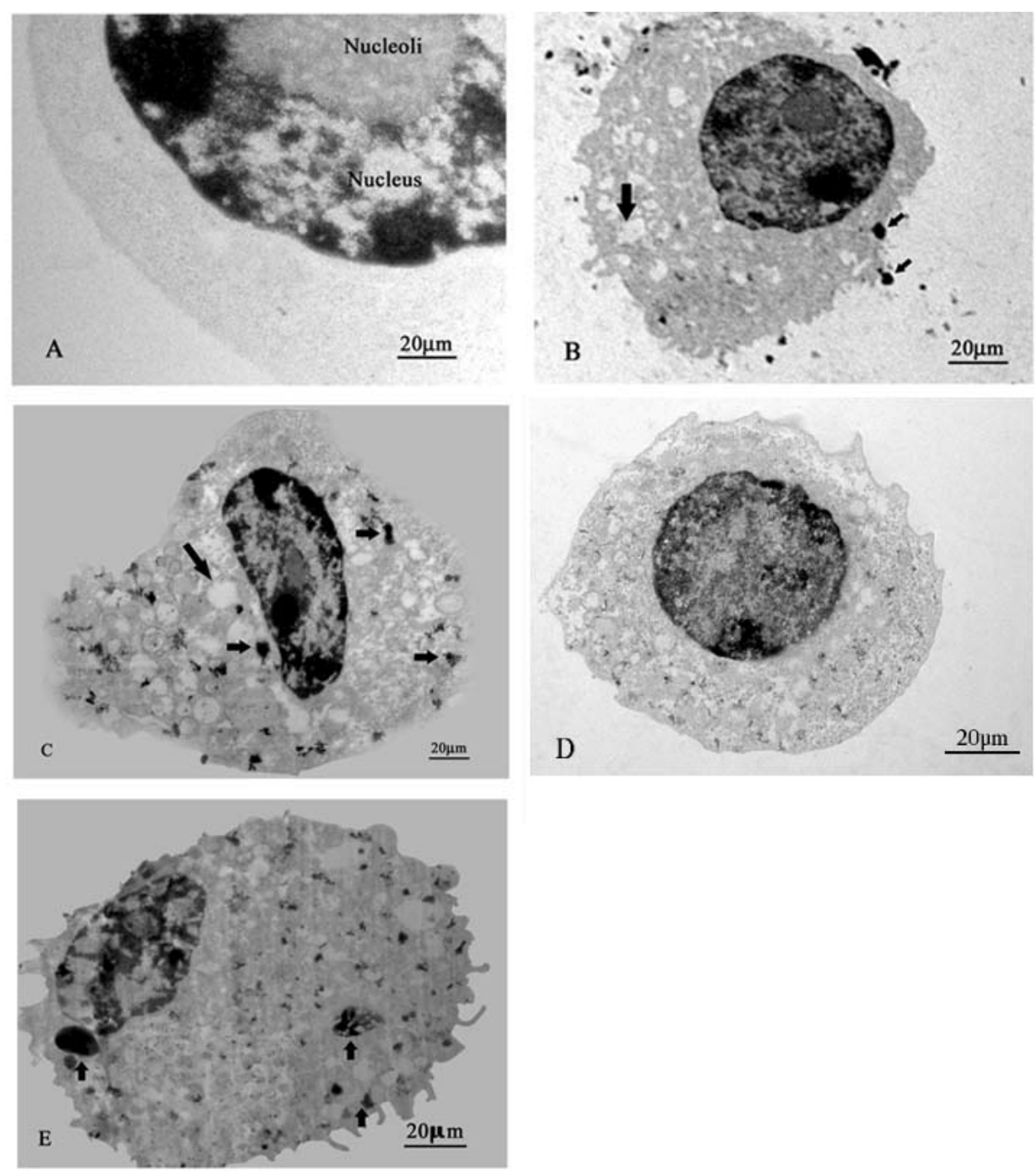

Fig.1: $\quad$ TEM photomicrographs of macrophages incubated with HA powder (arrow heads) for 7 min (B), 15 min (C), $30 \mathrm{~min}(\mathrm{D}) 60 \mathrm{~min}(\mathrm{E})$ and macrophage only (A)

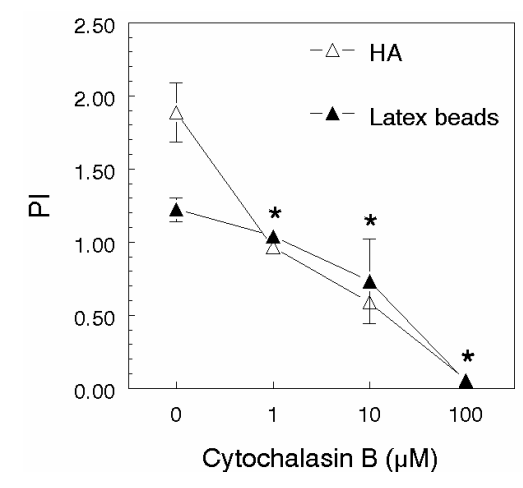

Fig. 2: The role of actin-filament polymerization in phagocytosis of HA particles and latex bead by RAW264.7 cells. The bar represents standard deviation. Asterisk $(*)$ indicates significance $(\mathrm{p}<0.05)$

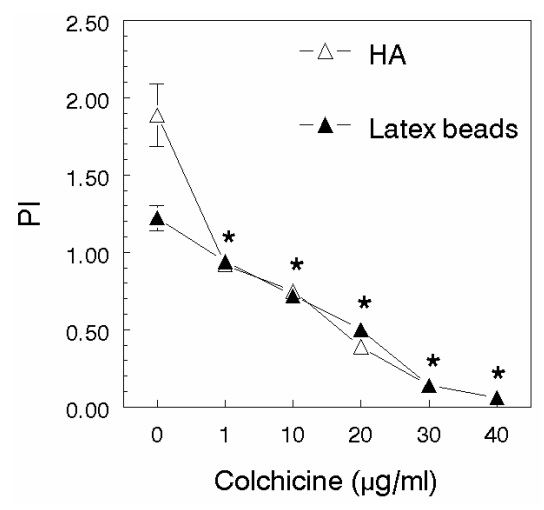

Fig. 3: The role of microtubule-disrupting agent in phagocytosis of HA particles and latex bead by RAW264.7 cells. The bar represents standard deviation. Asterisk (*) indicates significance $(\mathrm{p}<0.05)$ 
Most of the cells showed a high phagocytic activity at 15 min of incubation (Fig. 1C). The cells appeared with many HA particles with some cells showed exocytosis. Furthermore, HA-treated cells at 30 min appeared with multiple phagosome vacuoles showing homogenous distribution of HA particles (Fig.1D). Many vacuoles containing HA microcrystal precipitation were observed at $60 \mathrm{~min}$ (Fig. 1E).

Following pre-treatment of the cells with $1 \mu \mathrm{M}$ of Cytochalasin B or $1 \mu \mathrm{g}$ of colchicine, the numbers of ingested cells were considerably reduced as compared with untreated cells $(\mathrm{p}<0.05)$. Increased doses of Cytochalasin $\mathrm{B}$ or colchicine led to further reduction in the number of HA or latex bead ingested cells $(\mathrm{p}<0.05)$ (Fig. 2 and 3).

\section{DISCUSSION}

The results of the present study showed that murine macrophages phagocytosed HA particles at various incubation times under TEM as reveled by the presence of the particles in the cytoplasm and in the phagosome vacuoles. These results are in accordance with previous study showing that under TEM, HA particles were actively ingested by mice peritoneal macrophages ${ }^{[4]}$. Furthermore the HA particles were observed in side the phagolysosome as microcrystal. The reasons for this appearance might be due to the dissolution of the particles by the acidic environment in the cells ${ }^{[8]}$. Animal studies on dense HA implants in spongy bone showed the involvement of macrophages in phagocytosis of loose HA particles when examined under $\mathrm{TEM}^{[9]}$, thus confirming the above findings.

The present study demonstrated that the macrophage phagocytosis to HA was reduced in the presence of cytochalasin B, suggesting an actin polymerization-dependent phenomenon. During phagocytosis, the requirement of actin polymerization has also been shown when macrophages engulf particles such as bacteria ${ }^{[10]}$.

During macrophage phagocytic activities, microtubules are a key component of phagosome maturation $^{[11]}$. In this context, preincubation of RAW264.7 cells with microtubule disrupting agents, such as colchicine, decreased phagocytic activity of these cells to HA, suggesting a microtubule polymerization-dependent phenomenon. That microtubule disrupting agents inhibited polystyrene bead-stimulated murine macrophage phagocytosis ${ }^{[12]}$ highlights the results of the present study.

The extrapolation of the present study in humans implanted with HA remains speculative. Previous studies in animal models showed the presence of inflammatory cells such as macrophages adjacent to the implant materials and these cells ingested this material ${ }^{[5]}$. Rokkum et.al demonstrated that 14 out of 20 hip arthroplasties with HA-coated materials showed bone resorptive areas with some HA particles ingested by macrophages, suggesting that HA particles may induce phagocytic activities of macrophages in humans ${ }^{[13]}$. Therefore, the possibility of the dependence of phagocytic activities of macrophages to HA based implant materials in vivo on both actin and microtubule polymerization cannot be ruled out. However, further studies are needed to delineate this notion.

\section{CONCLUSION}

Murine macrophages ingested HA particles that were located in the vacuoles as observed under TEM. HA-induced phagocytic activities by murine macrophages were reduced by Cytochalasin B and colchicine, suggesting that HA induced phagocytosis by murine macrophages may be mediated by actincontaining microfilament system and microtubulebased motility systems.

\section{ACKNOWLEDGMENTS}

This research was supported by e-IRPA category EA grant, project No. 06-02-05-00029 EAR by the Malaysian Government. HA was prepared by School of Material and Mineral Resources Engineering, Universiti Sains Malaysia, Malaysia.

\section{REFERENCES}

1. LeGeros, R.Z., 2002. Properties of osteoconductive biomaterials: calcium phosphates. Clin. Orthop. Relat. Res., 395: 81-98.

2. Moreira-Gonzalez, A., I.T. Jackson, T. Miyawaki, V. DiNick and R. Yavuzer, 2003. Augmentation of the craniomaxillofacial region using porous hydroxyapatite granules. Plast. Reconstr. Surg., 111: 1808-17.

3. Hou, L.T., A.Y. Tsai, C.M. Liu and F. Feng, 2003. Autologous transplantation of gingival fibroblastlike cells and a hydroxylapatite complex graft in the treatment of periodontal osseous defects: cell cultivation and long-term report of cases. Cell.Transplant., 12:787-97.

4. Bosetti, M., V. Ottani, D. Kozel, M. Raspanti, V. De Pasquale, A. Ruggeri and M. Cannas, 1999. Structural and functional macrophages alterations by ceramics of different composition. Biomaterials, 20: 363-70.

5. Kawaguchi, H., T. Ogawa, M. Shirakawa, H. Okamoto and T. Akisaka 1992. Ultrastructural and ultracytochemical characteristics of multinucleated cells after hydroxyapatite implantation into rat periodontal tissue. J. Periodontal. Res., 27: 48-54.

6. Gopinath, V.K., M. Musa, A.R. Samsudin, P. Lalitha and W. Sosroseno, 2006. Role of Nitric oxide in Hydroxyapatite-induced phagocytosis by murine macrophage cell line (RAW264.7). Arch. Oral. Biol., 51: 339-344. 
7. Lang, D., F. Dohle, M. Terstesse, P. Bangen, C. August, H.G. Pauels and S. Heidenreich, 2002. Down-regulation of monocyte apoptosis by phagocytosis of platelets: involvement of a caspase-9, caspase-3 and heat shock protein 70dependent pathway. J. Immunol., 168: 6152-58.

8. Fukuchi, N., M. Akao and A. Sato, 1995. Effect of hydroxyapatite microcrystals on macrophage activity. Biomed. Mater. Eng., 5: 219-31.

9. Muller-Mai, C.M., C. Voigt and U. Gross, 1990. Incorporation and degradation of hydroxyapatite implants of different surface roughness and surface structure in bone. Scanning. Microsc., 4: 613-22.

10. Castellano, F., P. Chavrier and E. Caron, 2001. Actin dynamics during phagocytosis. Semin. Immunol., 13: 347-55.
11. Matteoni, R. and T.E. Kreis, 1987. Translocation and clustering of endosomes and lysosomes depends on microtubules. J. Cell. Biol., 105: 125365.

12. Toyohara, A. and K. Inaba, 1989. Transport of phagosomes in mouse peritoneal macrophages. J. Cell. Sci., 94: 143-53.

13. Rokkum, M., A. Reigstad and C.B. Johansson, 2002. HA particles can be released from well-fixed HA-coated stems: histopathology of biopsies from 20 hips 2-8 years after implantation. Acta. Orthop. Scand., 73: 298-306. 